\title{
A New Tensorial Framework for Single-Shell High Angular Resolution Diffusion Imaging
}

\author{
Luc Florack • Evgeniya Balmashnova - Laura Astola • \\ Ellen Brunenberg
}

Published online: 13 August 2010

(C) The Author(s) 2010. This article is published with open access at Springerlink.com

\begin{abstract}
Single-shell high angular resolution diffusion imaging data (HARDI) may be decomposed into a sum of eigenpolynomials of the Laplace-Beltrami operator on the unit sphere. The resulting representation combines the strengths hitherto offered by higher order tensor decomposition in a tensorial framework and spherical harmonic expansion in an analytical framework, but removes some of the conceptual weaknesses of either. In particular it admits analytically closed form expressions for Tikhonov regularization schemes and estimation of an orientation distribution function via the Funk-Radon Transform in tensorial form, which previously required recourse to spherical harmonic decomposition. As such it provides a natural point of departure for a Riemann-Finsler extension of the geometric approach towards tractography and connectivity analysis as has been stipulated in the context of diffusion tensor imaging (DTI), while at the same time retaining the natural coarse-
\end{abstract}

L. Florack ( $\varangle)$

Department of Mathematics \& Computer Science and

Department of Biomedical Engineering, Eindhoven University of Technology, Eindhoven, The Netherlands

e-mail: L.M.J.Florack@tue.nl

E. Balmashnova $\cdot$ L. Astola

Department of Mathematics \& Computer Science, Eindhoven

University of Technology, Eindhoven, The Netherlands

E. Balmashnova

e-mail: E.Balmashnova@tue.nl

L. Astola

e-mail: L.J.Astola@tue.nl

E. Brunenberg

Department of Biomedical Engineering, Eindhoven University

of Technology, Eindhoven, The Netherlands

e-mail: E.J.L.Brunenberg@tue.nl to-fine hierarchy intrinsic to spherical harmonic decomposition.

Keywords High angular resolution diffusion imaging . Diffusion tensor imaging - Tikhonov regularization . Orientation distribution function · Riemann-Finsler geometry

\section{Introduction}

High angular resolution diffusion imaging (HARDI) has become a standard MRI technique for mapping apparent water diffusion processes in fibrous tissues in vivo. The terminology HARDI is used here to collectively denote schemes that employ generic functions on the unit sphere, including Tuch's orientation distribution function (ODF) via classical Q-Ball imaging [44], the higher order diffusion tensor model and the diffusion orientation transform (DOT) by Özarslan et al. [36, 37], analytical Q-Ball imaging [14, 15], and the diffusion tensor distribution model by Jian et al. [27], et cetera.

A homogeneous polynomial expansion of HARDI data on the sphere has been proposed in a seminal paper by Özarslan and Mareci [36], which we shall refer to on several occasions further on. However, in this paper we consider an inhomogeneous expansion, including all (even) orders up to some fixed $N$ (including $N=\infty$ ), and exploit the redundancy of such a representation. The idea is to construct a polynomial series on the unit sphere in such a way that higher order terms capture residual degrees of freedom that cannot be represented by a lower order polynomial, akin to the hierarchical structure of a spherical harmonic expansion. (A different higher order tensor model for the unrestricted case, with a specific focus on the representation of 
the apparent diffusion coefficient (ADC), has been proposed by Liu et al. and Jensen et al. in terms of formal expansions in the $b$-parameter [26, 33].)

Interestingly, each homogeneous term in the new polynomial representation turns out to be self-similar under the Laplace-Beltrami operator on the unit sphere, mimicking the behaviour of its spherical harmonic equivalent. This self-similar nature is the fundamental property underlying many operations on HARDI that have hitherto been considered only in the context of a spherical harmonic decomposition, such as Tikhonov regularization and estimation of an orientation distribution function (ODF) from the ADC $[13,14,25,38]$. Regularization is of interest, since, unlike DTI [10, 11, 30], a generic HARDI model accounts for arbitrarily complex diffusivity profiles, thus raising the concomitant demand for regularization. ODFs are of interest for their putative connection to the complex architecture of fibrous tissue.

The higher order tensor representation introduced by Özarslan and Mareci (loc. cit.) does not exhibit selfsimilarity, making it somewhat problematic in a context of regularization or ODF estimation. (In this context, a function is called self-similar if it retains its form under regularization, except possibly for an overall attenuation factor.) The typical way to deal with this is to proceed via a spherical harmonic representation, which is inconvenient if the application context urges a tensorial approach, such as the geometric rationale towards tractography and connectivity analysis in classical DTI [4, 5, 16, 31, 38, 39]. Our polynomial representation can be seen as a formal refinement to the extent that degrees of freedom captured by a homogeneous $N$-th order tensor are decoupled and hierarchically rearranged into self-similar monomials of degrees up to $N$, obviating an explicit spherical harmonic detour altogether. (Spherical harmonics do play behind-the-scenes, as the span of our homogeneous terms will be seen to coincide with that of the spherical harmonics of corresponding order.)

\section{Theory}

We consider a higher order tensor representation of the form ${ }^{1}$

$u(y)=\sum_{k=0}^{\infty} u^{i_{1} \ldots i_{k}} y_{i_{1}} \ldots y_{i_{k}}$

\footnotetext{
${ }^{1}$ Summation convention applies to identical index pairs, i.e. whenever an identical index symbol occurs twice, once in lower and once in upper position, one tacitly sums over its possible values. Thus (1) is meant to be read as $u(y)=\sum_{k=0}^{\infty} \sum_{i_{1}=1}^{n} \ldots \sum_{i_{k}=1}^{n} u^{i_{1} \ldots i_{k}} y_{i_{1}} \ldots y_{i_{k}}$. For $k=0$ a product like $y_{i_{1}} \ldots y_{i_{k}}$ evaluates to unity by default, and a holor like $u^{i_{1} \ldots i_{k}}$ is to be understood as an index-free quantity, $u[35,42]$.
}

By $y^{i}$ and $y_{i}=\eta_{i j} y^{j}$ we denote the components of vectors (e.g. spatial directions), respectively dual covectors (e.g. normalized diffusion sensitizing gradients), confined to the unit sphere, and by $\eta_{i j}$ (respectively $\eta^{i j}$ ) the components of the Euclidean metric tensor (respectively its inverse) in the embedding 3-space. In Cartesian coordinates $\eta_{i j}=\eta^{i j}=1$ iff $i=j$, otherwise 0 . As outlined in the previous section $u(y)$ generically represents any HARDI-related scalar observable that can be represented as a function on the unit sphere. In typical cases in practice it is stipulated that

$u(y)=u(-y)$,

in which case only even orders will be of interest. This symmetry property, or equivalently the identification of antipodal points, $-y \sim y$, implies that $y$ actually represents orientation, not direction. We will only occasionally need this symmetry, in which case it will be explicitly indicated. Note that $u(y)$ represents a single codomain sample (e.g. signal strength as a function of direction/orientation at a fiducial point in space), its dependence on the spatial domain variable $x \in \mathbb{R}^{3}$ is suppressed in the notation.

The collection of polynomials on the sphere,

$\mathcal{B}=\bigcup_{k \in \mathbb{Z}_{0}^{+}} \mathcal{B}_{k} \quad$ with $\mathcal{B}_{k}=\left\{y_{i_{1}} \ldots y_{i_{k}} \mid k \in \mathbb{Z}_{0}^{+}\right\}$,

is complete, but redundant. Apart from the fact that odd orders might be of no interest, redundancy is evident from the fact that lower order even/odd monomials can be reproduced from higher order ones of equal parity through contractions as a result of $\eta^{i j} y_{i} y_{j}=1$ (unit sphere confinement or "single-shell" restriction). Thus any monomial of order $k \leq N \in \mathbb{Z}_{0}^{+}$is linearly dependent on the set of $N$-th order monomials of equal parity. This, of course, justifies the approach by Özarslan and Mareci, in which data are fitted against linear combinations of $N$-th order monomials, discarding all lower order terms.

However, exploiting the redundancy of $\mathcal{B}$, (3), we may choose to encode residual information in higher order tensor coefficients. As $N \rightarrow \infty$ this residual tends to zero, while all established tensor coefficients of ranks lower than $N$ remain fixed in the process of incrementing $N$. The coefficients are constructed as follows. Suppose we are in possession of $u^{i_{1} \ldots i_{k}}$ for all $k=0, \ldots, N-1$, then we consider the function

$E_{N}\left(u^{j_{1} \ldots j_{N}}\right)=\int\left(u(y)-\sum_{k=0}^{N} u^{i_{1} \ldots i_{k}} y_{i_{1}} \ldots y_{i_{k}}\right)^{2} d y$

to find the $N$-th order coefficients by minimization (integration here and henceforth takes place over the unit sphere). Setting

$$
\frac{\partial E_{N}\left(u^{j_{1} \ldots j_{N}}\right)}{\partial u^{i_{1} \ldots i_{N}}}=0
$$


one obtains the following linear system for the contravariant tensor coefficients $u^{i_{1} \ldots i_{N}}$ :

$$
\begin{aligned}
& A_{i_{1} \ldots i_{N} j_{1} \ldots j_{N}} u^{j_{1} \ldots j_{N}} \\
& \quad=\int u(y) y_{i_{1}} \ldots y_{i_{N}} d y-\sum_{k=0}^{N-1} A_{i_{1} \ldots i_{N} j_{1} \ldots j_{k}} u^{j_{1} \ldots j_{k}},
\end{aligned}
$$

with symmetric covariant tensor coefficients

$$
A_{i_{1} \ldots i_{k}}=\int y_{i_{1}} \ldots y_{i_{k}} d y \text {. }
$$

Note that the second inhomogeneous term on the r.h.s. of (6) is absent in the scheme proposed by Özarslan and Mareci. By symmetry considerations constant odd-rank tensors vanish:

$$
A_{i_{1} \ldots i_{2 k+1}}=0 \quad\left(k \in \mathbb{Z}_{0}^{+}\right)
$$

All even-rank constant tensors must be products of the Euclidean metric tensor, so we stipulate

$$
A_{i_{1} \ldots i_{2 k}}=\gamma_{k} \eta_{\left(i_{1} i_{2}\right.} \ldots \eta_{\left.i_{2 k-1} i_{2 k}\right)}
$$

for some constant $\gamma_{k}$. Parentheses symbolize index symmetrization, i.e. if $T_{i_{1} \ldots i_{p}}$ is a rank- $p$ tensor, then

$T_{\left(i_{1} \ldots i_{p}\right)}=\frac{1}{p !} \sum_{\pi} T_{\pi\left(i_{1} \ldots i_{p}\right)}$

in which the summation runs over all $p$ ! permutations $\pi$ of index positions. The constant $\gamma_{k}$ needs to be determined for each $k \in \mathbb{Z}_{0}^{+}$. One way to find $\gamma_{k}$ is to evaluate (8) for $i_{1}=\cdots=i_{2 k}=1$ in a Cartesian coordinate system, since the symmetric product of metric tensors on the r.h.s. evaluates to 1 for this case:

$\gamma_{k}=A_{1 \ldots \leftarrow 2 k \text { indices } \rightarrow \ldots 1}=\int y_{1}^{2 k} d y$.

This is a special case of the closed-form multi-index representation by Folland [21] and Johnston [28]:

$$
\int y_{1}^{\alpha_{1}} \ldots y_{n}^{\alpha_{n}} d y=\frac{2}{\Gamma\left(\frac{1}{2}|\alpha|+\frac{n}{2}\right)} \prod_{i=1}^{n} \Gamma\left(\frac{1}{2} \alpha_{i}+\frac{1}{2}\right),
$$

if all $\alpha_{j}$ are even (otherwise the integral vanishes). Here $|\alpha|=\alpha_{1}+\cdots+\alpha_{n}=2 k$ denotes the norm of the multiindex, and

$$
\Gamma(t)=\int_{0}^{\infty} s^{t-1} e^{-s} d s
$$

is the gamma function. Relevant properties: $\Gamma(\ell)=(\ell-1)$ ! and $\Gamma\left(\ell+\frac{1}{2}\right)=\left(\ell-\frac{1}{2}\right) \ldots \frac{1}{2} \sqrt{\pi}=(2 \ell) ! \sqrt{\pi} /\left(4^{\ell} \ell !\right)$ for $\ell \in \mathbb{Z}_{0}^{+}$.
From (6) and (7-8) it follows that, for general spatial dimension $n$,

$$
A_{i_{1} \ldots i_{2 k}}=\frac{2 \Gamma\left(k+\frac{1}{2}\right) \Gamma\left(\frac{1}{2}\right)^{n-1}}{\Gamma\left(k+\frac{n}{2}\right)} \eta_{\left(i_{1} i_{2}\right.} \ldots \eta_{\left.i_{2 k-1} i_{2 k}\right)} .
$$

Equations (7) and (13) form the tensorial counterpart of (11). Note that the coefficients $A_{i_{1} \ldots i_{k}}$ are independent of the physical interpretation of the expansion on the r.h.s. of (1) as long as its construction is based on an energy minimization principle of the form (4) for the observable of interest.

The interesting property of our inhomogeneous expansion, viz. that it realizes a (partial) hierarchical ordering of degrees of freedom akin to the one induced by spherical harmonic decomposition, is manifest in the following observation. Consider the Laplace-Beltrami operator $\Delta$ on the unit sphere, then for any $N \in \mathbb{Z}_{0}^{+} \cup\{\infty\}$ and $t>0$,

$u_{N}(y, t) \equiv e^{t \Delta} u_{N}(y)=\sum_{k=0}^{N} u^{i_{1} \ldots i_{k}}(t) y_{i_{1}} \ldots y_{i_{k}}$,

with

$u^{i_{1} \ldots i_{k}}(t)=e^{-k(k+1) t} u^{i_{1} \ldots i_{k}}$.

A proof of (14-15) is provided elsewhere [17], where it is shown that the span of the homogeneous polynomials $u^{i_{1} \ldots i_{k}} y_{i_{1}} \ldots y_{i_{k}}$ for fixed $k$ coincides with that of the spherical harmonics $Y_{k m}(y)$ (with $m=-k,-k+1, \ldots$, $k-1, k)$ of the same order, and hence constitutes a degenerate eigenspace of the Laplace-Beltrami operator with eigenvalue $-k(k+1)$. (It is understood, in this case, that $n=3$, and that the coordinates $y \in \mathbb{R}^{3}$ of the embedded unit sphere are parameterized in terms of the usual spherical coordinates $\theta, \phi$.$) This is nontrivial, since the monomials$ $y_{i_{1}} \ldots y_{i_{k}}$ themselves are not eigenfunctions of $\Delta$. Indeed, (14-15) show that the degrees of freedom in the polynomial expansion are segregated in such a way that we may interpret each homogeneous term as an incremental refinement of detail relative to that of the lower order expansion. To appreciate the significance of (14), note that $u_{N}(y, t)$ satisfies the heat equation on the unit sphere:

$\frac{\partial u}{\partial t}=\frac{1}{\sqrt{|g|}} \partial_{\mu}\left(g^{\mu \nu} \sqrt{|g|} \partial_{\nu} u\right)=\Delta u$

in which the initial condition corresponds to the $N$-th order expansion of the raw data, $u_{N}(y, 0)=u_{N}(y)$, and $|g|=$ $\operatorname{det} g_{\mu \nu}$. Here the Riemannian metric of the embedded unit sphere is given by

$g_{\mu \nu}=\frac{\partial y^{i}}{\partial \xi^{\mu}} \eta_{i j} \frac{\partial y^{j}}{\partial \xi^{v}}$ 
in which $\xi^{\mu}(\mu=1, \ldots, n-1)$ parameterize the unit sphere in $\mathbb{R}^{n}$. It is evident from the above that the linear combinations $u^{i_{1} \ldots i_{k}} y_{i_{1}} \ldots y_{i_{k}}$, unlike the monomials $y_{i_{1}} \ldots y_{i_{k}}$ themselves, are eigenfunctions of the heat operator $\exp (t \Delta)$, i.e. self-similar polynomials on the unit sphere, with eigenvalues $e^{-k(k+1) t}$. Recall that the heat operator can be seen as the canonical resolution degrading ${ }^{2}$ semigroup operator [29]. The parameter $t$ captures inverse angular resolution at which the raw HARDI data are resolved (at a fixed point in space). Indeed, (14) shows that each homogeneous polynomial ( $k$ fixed) retains its form upon blurring (increasing $t$ ), up to a $t$-dependent attenuation factor $e^{-k(k+1) t}$. This characteristic decay is analogous to the $e^{-t\|\omega\|^{2}}$-attenuation of a Gaussian blurred image in the Euclidean frequency domain, with frequency coordinate $\omega \in \mathbb{R}^{n}$. Cf. Descoteaux et al. [14] and Hess et al. [25] for similar regularization schemes expressed relative to a spherical harmonic basis, and to Florack et al. [18] for a comparison between the spherical harmonic and tensorial approaches towards regularization.

\section{Examples}

We consider some examples of possible application contexts for the generic theory presented in the previous section.

\subsection{Relation to Higher Order DTI and Regularization}

In this example we consider the Stejskal-Tanner equation [43],

$S(y)=S_{0} \exp (-b D(y))$,

and identify the generic function $u(y)$ from the previous theory with the ADC $D(y)$, considered as a function of orientation (in particular, $D(y)=D(-y)$ ).

Here are some examples of (13) for the relevant case, $n=3$ :

$k=0: \quad A=4 \pi$,

$k=1: \quad A_{i j}=\frac{4 \pi}{3} \eta_{i j}$,

$k=2: \quad A_{i j k \ell}=\frac{4 \pi}{15}\left(\eta_{i j} \eta_{k \ell}+\eta_{i k} \eta_{j \ell}+\eta_{i \ell} \eta_{j k}\right)$.

The corresponding linear systems, (6), are as follows:

$$
\begin{aligned}
& A D=\int D(y) d y, \\
& A_{i j} D^{j}=\int D(y) y_{i} d y-A_{i} D, \\
& A_{i j k \ell} D^{k \ell}=\int D(y) y_{i} y_{j} d y-A_{i j} D-A_{i j k} D^{k} .
\end{aligned}
$$

\footnotetext{
2“Degrading" is meant in a sense akin to cartographic generalization, i.e. without negative connotation.
}

It follows that the scalar constant $D$ is just the average diffusivity:

$D=\frac{\int D(y) d y}{\int d y}$.

The constant vector $D^{i}$ vanishes identically, as it should (since no basis independent constant vectors exist). For the rank-2 tensor coefficients we find the traceless matrix

$D_{i j}=\frac{15 \int D(y) y_{i} y_{j} d y-5 \int D(y) d y \eta_{i j}}{2 \int d y}$,

and so forth. If, instead, we fit a homogeneous second order polynomial to the data (by formally omitting the $\sum$-terms on the r.h.s. of (6)), as proposed by Özarslan and Mareci, we obtain the following rank-2 tensor coefficients:

$D_{i j}^{\ddot{O ̈ . M .}}=\frac{15 \int D(y) y_{i} y_{j} d y-3 \int D(y) d y \eta_{i j}}{2 \int d y}$,

which are clearly different. However, one may observe that the full second order expansions coincide. The difference in coefficients, in this example, is explained by the contribution already contained in the lowest order term of our polynomial, which in Özarslan and Mareci's scheme has migrated to the second order tensor.

In fact, we conjecture that equality holds to any order $N$. More precisely, if $D_{N}(y)$ denotes the truncated expansion of (1) including monomials of orders $k \leq N$ only, with coefficients constructed according to $(6-13), D_{N}^{\text {O.M. }}(y)$ the $N$-th order homogeneous polynomial expansion proposed by Özarslan and Mareci, and $D_{N}^{\text {S.H. }}(y)$ the canonical spherical harmonic decomposition, cf. Frank [22] and Alexander et al. [1], then

$D_{N}(y)=D_{N}^{\text {O.M. }}(y)=D_{N}^{\text {S.H. }}(y)$.

(The formal limit $N \rightarrow \infty$ makes sense only for left and right hand sides.) This shows the equivalence of all three representations.

In particular (14) reveals that the classical rank-2 DTI representation, defined via the Stejskal-Tanner formula, (18), arises not merely as an approximation under the assumption that the apparent diffusion coefficient can be written as

$D(y) \approx D_{\text {DTI }}(y)=D_{\text {DTI }}^{i j} y_{i} y_{j}$,

but expresses the exact asymptotic behaviour of $D(y, t)=$ $D_{\infty}(y, t)$ as $t \rightarrow \infty$, recall (14):

$$
D(y, t)=\underbrace{\left(D \eta^{i j}+e^{-6 t} D^{i j}\right) y_{i} y_{j}}_{D_{\mathrm{DTI}}(y, t)=D_{\mathrm{DTI}}^{i j}(t) y_{i} y_{j}}+\mathcal{O}\left(e^{-20 t}\right) .
$$


Thus the DTI tensor is not self-similar, but has a bimodal resolution dependence. The actual limit of vanishing resolution is of course given by a complete averaging over the sphere:

$\lim _{t \rightarrow \infty} D(y, t)=\lim _{t \rightarrow \infty} D_{\mathrm{DTI}}(y, t)=D$,

recall (19).

The generalized counterpart of (24) suggests a Finsler [6, 7, 34] rather than Riemannian [5, 16, 31, 32, 38, 39] framework for tractography and connectivity analysis, whereby one replaces the contravariant rank-2 (dual metric) tensor $D_{\text {DTI }}(y, t)$ by

$D_{\mathrm{HARDI}}^{i j}(y, t)=D_{\mathrm{DTI}}^{i j}(t)+\sum_{k=1}^{\infty} D^{i_{1} \ldots i_{k} i j}(t) y_{i_{1}} \ldots y_{i_{k}}$,

with multimodal additional $y$-dependent terms as defined in (14). For details on Finsler geometry the books by Bao et al. [8] and Shen [41] are highly recommended. Literature on Riemannian geometry is abundant. Spivak [42] and Misner et al. [35] are classics. Rund [40] provides a useful variational perspective.

\subsection{Derivation of an ODF via the Funk-Radon Transform}

In the second example we identify the function $u(y)$ from Sect. 2 with a raw HARDI signal (again at an implicitly defined spatial locus $x \in \mathbb{R}^{3}$ ). The precise way of identification will become clear soon.

To begin with, we consider the hypothetical signal function $S(q)$ defined for all Euclidean diffusion wave vectors $q \in \mathbb{R}^{3}$, and recall its relation to the diffusion probability function $P(r)$, with Euclidean displacement vector $r \in \mathbb{R}^{3}$, via Fourier transformation:

$P(r)=\int_{\mathbb{R}^{3}} S(q) e^{2 \pi i r \cdot q} d q$.

Considerations of signal-to-noise ratio and acquisition time have led Tuch [44] to introduce the single-shell orientation diffusion function (ODF), defined on the unit sphere $\|y\|=1$,

$\Psi(y)=\int_{0}^{\infty} P(\lambda y) d \lambda$,

and to consider an approximation $\Psi_{Q}(y) \approx \Psi(y)$ in the form of the so-called extended Funk-Radon transform in terms of a single-shell acquisition in $q$-space:

$\Psi_{Q}(y)=\int_{\mathbb{R}^{3}} S(q) \delta(q \cdot y) \delta(\|q\|-Q) d q$,

in which $Q>0$ denotes the radius of the acquisition shell in $q$-space. This " $Q$-ball" reconstruction has gained popularity because of the data acquisition efficiency of single-shell recordings and for its robustness (for not too large $Q$ ).
There exist similar analytical approaches towards Q-ball imaging, cf. Descoteaux et al. [14], Anderson [2], and Hess et al. [25], all of which exploit spherical harmonic decomposition. We focus on the approach by Descoteaux et al. based on the so-called Funk-Hecke theorem, which yields for any spherical harmonic function $Y_{\ell m}(y)$ of capital order $\ell$

$\int_{\|\eta\|=1} \delta(y \cdot \eta) Y_{\ell m}(\eta) d \eta=2 \pi Y_{\ell m}(y) P_{\ell}(0)$

in which $P_{\ell}(t)$ denotes the Legendre polynomial of degree $\ell$. Note that for even $\ell=2 k$ we have

$P_{2 k}(0)=\frac{(-1)^{k}(2 k) !}{2^{2 k}(k !)^{2}}=\frac{(-1)^{k}}{\sqrt{\pi}} \frac{\Gamma\left(k+\frac{1}{2}\right)}{\Gamma(k+1)}$.

Taking into account that the homogeneous polynomials, i.e. the terms in (1) and (14) with $k$ fixed, belong to the span of the spherical harmonics of the same order $k$, (29) and (30) allow us to write the ODF in tensor representation with tensorial coefficients $S^{i_{1} \ldots i_{k}}$ induced by the signal $S=S_{\infty}$,

$S(y)=\sum_{k=0}^{\infty} S^{i_{1} \ldots i_{k}} y_{i_{1}} \ldots y_{i_{k}}$

according to the general recursive scheme given by (6):

$\Psi(y)=2 \pi \sum_{k=0}^{\infty} P_{k}(0) S^{i_{1} \ldots i_{k}} y_{i_{1}} \ldots y_{i_{k}}$.

Analogous to (14) we may incorporate a regularization parameter via a suitable generator based on the Laplace-Beltrami operator on the unit sphere, e.g.

$\Psi(y, t)=2 \pi \sum_{k=0}^{\infty} P_{k}(0) S^{i_{1} \ldots i_{k}}(t) y_{i_{1}} \ldots y_{i_{k}}$,

with $t$-dependent attenuation of coefficients, recall (15). (Truncation at finite order $N$ results in an additional regularization effect.)

In particular, the example shows that no explicit detour via a spherical harmonic representation is needed to achieve a closed-form analytical Q-ball representation within a tensorial framework. The resulting diffusion ODF is equivalent to the one obtained from the established analytical Q-ball algorithm in the spherical harmonic basis. This is of interest in Q-ball applications where the tensor formalism is the preferred choice, such as in a differential geometric (notably Finslerian) approach towards tractography and connectivity analysis.

\subsection{Spatial Regularization}

Realizing that the unit-sphere function and its coefficients introduced in (14-15) also depend on a spatial variable 
$x \in \mathbb{R}^{3}$, we may consider to combine regularization in the spatial domain (with a control parameter $s>0$ for spatial coherence, cf. Assemlal et al., Chen et al. and Goh et al. $[3,12,24]$ ) with codomain regularization (with the independent parameter $t>0$ for signal smoothing at fixed position),

$$
\begin{aligned}
u_{N}(x, y, s, t) & =e^{s \Delta_{x}} e^{t \Delta_{y}} u_{N}(x, y) \\
& =\sum_{k=0}^{N} u^{i_{1} \ldots i_{k}}(x, s, t) y_{i_{1}} \ldots y_{i_{k}},
\end{aligned}
$$

in which $\Delta_{x}$ is the standard Laplacian for Euclidean 3space, and $\Delta_{y}$ the one appropriate for the unit sphere ( $\Delta$ in the foregoing). For example, on an unbounded domain the operator $e^{s \Delta_{x}}$ can be identified (in spatial representation) as Gaussian convolution:

$u^{i_{1} \ldots i_{k}}(x, s, t)=e^{-k(k+1) t}\left(u^{i_{1} \ldots i_{k}} * \phi_{s}\right)(x)$,

in which

$\phi_{s}(x)=\frac{1}{\sqrt{4 \pi s}^{3}} \exp \left(-\frac{\|x\|^{2}}{4 s}\right)$.

Other types of regularization and domain boundary restrictions can be straightforwardly accounted for. In particular this combined domain-codomain regularization can be applied to the polynomial representations of the spatially extended ADC function $D(x, y)$ from Sect. 3.1 and signal function $S(x, y)$ from Sect. 3.2.

As a special case, recall the Q-Ball representation of (34) and consider it as a function of position in Euclidean space $\mathbb{R}^{n}$, then we may write the doubly-regularized ODF as

$$
\begin{aligned}
\Psi(x, y, s, t)= & \frac{1}{(2 \pi)^{n}} \sum_{k=0}^{\infty} \int_{\mathbb{R}^{n}} e^{i \omega \cdot x} e^{-s\|\omega\|^{2}} e^{-k(k+1) t} \\
& \times \hat{\Psi}^{i_{1} \ldots i_{k}}(\omega) y_{i_{1}} \ldots y_{i_{k}} d \omega,
\end{aligned}
$$

with spatial Fourier coefficients

$\hat{\Psi}^{i_{1} \ldots i_{k}}(\omega)=\int_{\mathbb{R}^{n}} e^{-i \omega \cdot x} \Psi^{i_{1} \ldots i_{k}}(x) d x$,

and, in terms of the coefficients of the actual raw signal,

$\Psi^{i_{1} \ldots i_{k}}(x)=2 \pi P_{k}(0) S^{i_{1} \ldots i_{k}}(x)$.

This double-frequency representation, (38-40), reveals the effect of simultaneous regularization in the (infinite) spatial domain and on the (compact) unit sphere as a highfrequency attenuation process (with continuous, respectively discrete frequency spectrum for $\omega$ and $k$ ). Of course, an equivalent representation can be obtained using the spherical harmonic basis $\left\{Y_{k m} \mid k=0,1,2, \ldots ;-k \leq m \leq k\right\}$ instead of the redundant set $\mathscr{B}$ of (3).
In the Riemann geometric rationale the (pointwise) inverse of the contravariant diffusion tensor image is identified with the (covariant) metric tensor. In the Finsler geometric rationale one may instead stipulate a Finsler norm function $F(y)$ as a generalization of a Riemannian metric induced norm $\sqrt{g_{i j} y^{i} y^{j}}$, so that the corresponding (regularized) dual Finsler metric is given by

$g^{i j}(x, y, s, t)=\frac{\partial^{2} \Psi(x, y, s, t)}{\partial y_{i} \partial y_{j}}$.

By definition (ignoring our control parameters for regularization)

$g_{i j}(x, y)=\frac{\partial^{2} F^{2}(x, y)}{\partial y^{i} \partial y^{j}}$,

is called the (covariant) Finsler metric, and so we have the following relation with the ODF, recall (38):

$\frac{\partial^{2} \Psi(x, y, s, t)}{\partial y_{i} \partial y_{k}} \frac{\partial^{2} F^{2}(x, y, s, t)}{\partial y^{k} \partial y^{j}}=\delta_{i}^{j}$.

We stipulate this as our conjecture for a rigorous RiemannFinsler geometric approach towards tractography and connectivity analysis.

To actually prove this conjecture beyond heuristics a more thorough investigation of this "correspondence principle" will need to be conducted. In any case, the interesting feature of any Finsler metric, such as (41), is its directional dependence, i.e. its dependence on $y$, which distinguishes it from a Riemannian metric. The latter arises as a special case, either as the hypothetical case in which all coefficients of orders $k>2$ happen to vanish identically, or, in an operational sense, in the asymptotic limit of large regularization $t \gg 0$ in the signal codomain akin to (24). We refer to the literature for details on Riemann-Finsler geometry [8].

Figure 1 shows a synthetic example of a single-shell signal, illustrating the effect of combined domain and codomain regularizations. The example was created using a Gaussian mixture model:

$S=\frac{1}{2} S_{0}\left(e^{-b g^{T} D_{1} g}+e^{-b g^{T} D_{2} g}\right)$,

in which $D_{1}$ and $D_{2}$ are linear tensors with principal eigenvectors oriented along stipulated fibers, and eigenvalues $(3,3,17) \times 10^{-4} \mathrm{~mm}^{2} / \mathrm{s}$. The b-value is $1000 \mathrm{~s} / \mathrm{mm}^{2}$ and the number of gradient directions 80 .

The glyphs in the first column of Fig. 1 vary from top to bottom due to spatial averaging only (a Gaussian scale space process), i.e. no regularization is applied to their local profiles. On the other hand, going from left to right, we observe a simplification of these profiles that is exclusively driven by pointwise regularization in the signal domain, i.e. 
Fig. 1 Artificial data of crossing fibers built with a Gaussian mixture model, and perturbed by Rician noise with $\mathrm{SNR}=15.3$. From left to right: signal (codomain) regularization, with $t=0.00,0.018,0.050,0.14$. From top to bottom: spatial (domain) regularization, with $s=0.00,0.22,0.37,0.61$. Recall (35). Cf. text for explanations

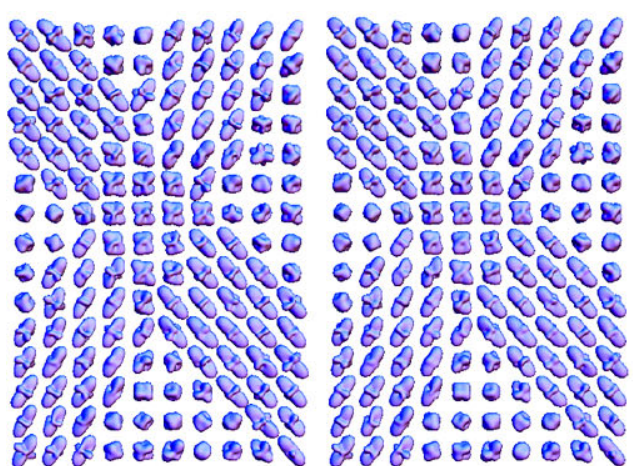

00000000000000000000

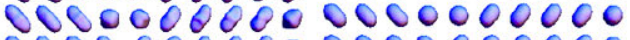
coberoodo.0000000000 00000000000000000000 00080000000000000000 00080E00000000000000 00080080000000000000 00000000000000000000 - 000000000000000000 00000000000000000000 20000000000000000000 80000000000000000000 00000000000000000000 00000000000000000000 00000000000000000000
0000000000 000000000 0,0000000 $\operatorname{sic} 000000$ 060000000 080880000 -

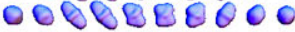
OOOBEEEOCO $00008 E$ CDO - O00008c8 $00 \theta \theta 008$

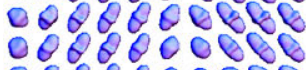
QOQQ00008R
0000000000 0000000000 0000000000 000000000 0000000000 - c0000000 - cocorogro - OOOCER000 DOOCER8000 0000 rese 00000000000000000000 1 00000000000000000000 - Q08000808 00000000000000000000 000800000000000000000000000000 000800000000000000000000000000

00000000000000000000 00000000000000000000 00000000000000000000 00000000000000000000 00000000000000000000 00000000000000000000 00000000000000000000 00000000000000000000 00000000000000000000 00000000000000000000
00000000000000000000 000000000000000000000000000000 000000000000000000000000000000 008000000000000000000000000000 000600000000000000000000000000 080800000000000000000000000000 - coberob0000000000000000000000 000000000000000000000000000000 000088000000000000000000000000 000008800000000000000000000000 000000008000000000000000000000 $00080006 \cos 000000000000000000$ 008000086000000000000000000000 000000000000000000000000000000 000000000000000000000000000000

0000000000000000000000000000000000000000 0000000000000000000000000000000000000000 0800000000000000000000000000000000000000 cebeco0000 000000000000000000000000000000

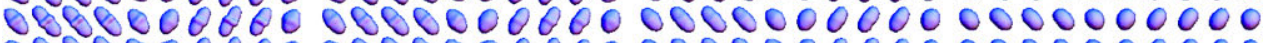
$0858 B 0 \theta 000000000000000000000000000000000$ $008 \mathrm{c} \theta 0000000000000000000000000000000000$ 0000880000000000000000000000000000000000 $0008 B 88000000080600000000000000000000000$

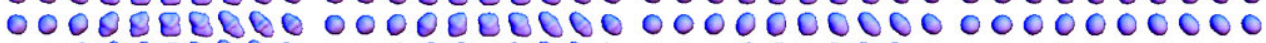
- 00000800 000000000000000000000000000000

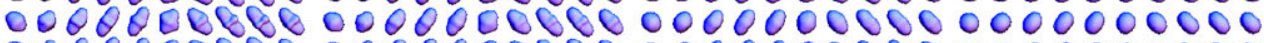
$0 Q \theta Q Q 00800008000008000000000000000000000$ 0000000000000000000000000000000000000000 00000000RR 000000000000000000000000000000

a parallel process involving no spatial neighbourhood interactions. In general one will need to incorporate both types of regularization for reasons of robustness and depending on data and objective. It is not a priori self-evident that a single pair of parameters ( $s, t)$ ("scale selection") may work in practical applications, although it is an obvious first step towards more sophisticated attempts to solve the notorious problem of scale ("deep structure"), e.g. through a coarseto-fine approach. Even in the one-parameter case this is still an outstanding problem [20, 29].

Figure 2 shows an RGB map representing the principal diffusion directions (main eigenvector of DTI tensor) in a Wistar rat brain (red: left-right, green: anterior-posterior, 
Fig. 2 Brain of 17-week old male Wistar rat, and region of interest
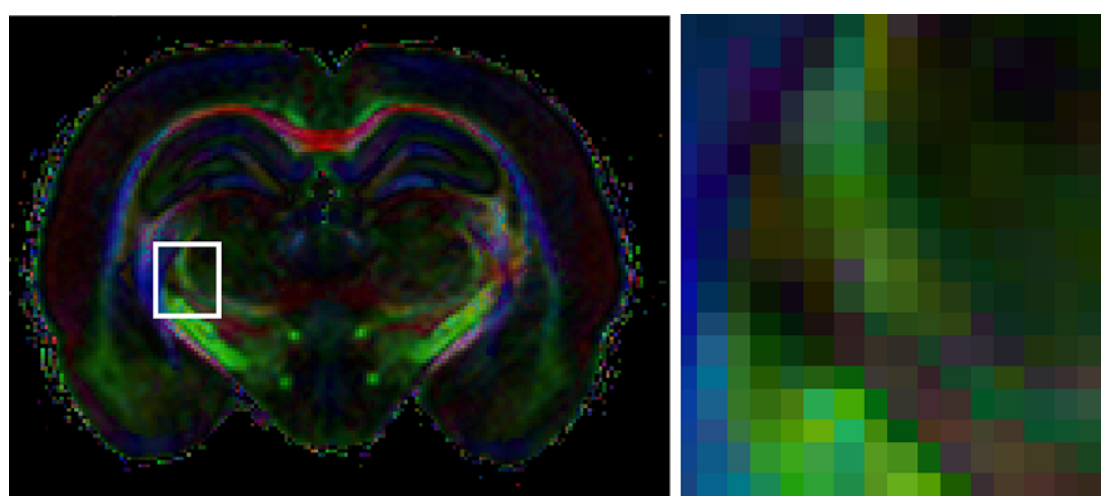

blue: superior-inferior). The luminance is weighted by the fractional anisotropy value.

The rat brain data were measured on a 9.4T Bruker Biospec AVANCE-III system. HARDI was acquired using a diffusion-weighted spin-echo sequence with two unipolar pulsed field gradients placed symmetrically around the 180 degree pulse (TE 27 ms, TR 4000 ms, NA 1, total time 19 hours). Fifteen coronal slices with slice thickness $500 \mu \mathrm{m}$ and interslice gap $50 \mu \mathrm{m}$ were measured. The matrix size was $128 \times 128$, zero-filled to $256 \times 256$ pixels. The FOV was $25.6 \times 25.6 \mathrm{~mm}^{2}$, leading to an in-plane pixel dimension of $100 \mu \mathrm{m}$. A series of 54 images with different gradient directions and b-value $3000 \mathrm{~s} / \mathrm{mm}^{2}$, together with an unweighted image, was measured.

The enlarged $\mathrm{ROI}^{3}$ on the right-hand side in Fig. 2 focuses on the junction of the internal capsule (large white matter bundle, represented as a bright green structure, partly seen in the lower left corner of the ROI) and the zona incerta (gray matter, lying just above the internal capsule). In the zona incerta, many small fiber bundles are intermingled, causing crossings to occur in the HARDI data. These fiber bundles include tracts from the subthalamic nucleus to more lateral nuclei such as the globus pallidus.

Figure 3 shows the combined (three-dimensional) regularization in domain and codomain for the ROI. Again, notice that the apparent smoothing effects visible in the glyph representations have various causes. On the top row smoothing is entirely due to individual glyph regularization (lowering of angular resolution). In the left column smoothing of glyphs is caused by Gaussian weighted spatial averaging only (lowering of spatial resolution). For tractography purposes one may want to control both spatial and angular resolution depending on the size of the structures of interest, signal-to-noise ratio, robustness, et cetera.

\footnotetext{
${ }^{3}$ In human subjects, proper fiber tracking in a similar region might be important for neurosurgical purposes such as the planning of electrode implantation for deep brain stimulation of the subthalamic nucleus.
}

\section{Conclusion and Recommendations for Future Work}

We have posited a tensorial decomposition of HARDIrelated functions on the unit sphere in terms of inhomogeneous polynomials. The resulting polynomial representation may be regarded as the tensorial counterpart of the canonical spherical harmonic decomposition. Although formally equivalent to the homogeneous polynomial expansion proposed by Özarslan and Mareci [36], our inhomogeneous expansion differs in an essential way. It segregates the HARDI degrees of freedom into a hierarchy of homogeneous polynomials that are self-similar under the act of resolution degradation induced by the heat operator, $\exp (t \Delta)$, or similar Tikhonov regularization operators of the type $f(t \Delta)$, each with a characteristic decay that depends on order (for fixed $t \in \mathbb{R}^{+}$).

The polynomial framework is generically applicable to single-shell representations. In the context of HARDI we have illustrated its potential use by several examples. One is the well-posed extension of rank-2 DTI to arbitrary ranks while simultaneously controlling regularity via a control parameter $t>0$, exploiting the special properties of the homogeneous terms. The asymptotic case of almost vanishing resolution $(t \rightarrow \infty)$ reproduces the diffusion tensor of classical diffusion tensor imaging (DTI), with one constant and one resolution-dependent mode. Whereas this example is based on the Stejksal-Tanner equation for the ADC, a second example involves only the HARDI signal itself, and mimics the popular analytical Q-ball rationale within the tensorial framework, again by virtue of the special properties of the homogeneous terms in the polynomial expansion. A third example shows how the representations can be simultaneously regularized in spatial and signal domains, introducing a second regularization parameter $s>0$ (scale) for spatial coherence. A suggestion for future research is to consider regularization in domain and/or codomain, (35), for sufficiently small negative values of $s$ and $t$, in combination with truncation at some optimal order $N(s, t)$ for the purpose of enhancement (inverse diffusion). Truncation makes this scenario well-posed, but amplification of noise 
Fig. 3 Recall Fig. 2, and (35). From left to right: signal (codomain) regularization, with $t=0.00,0.0025,0.018,0.14$.

From top to bottom: spatial (domain) regularization, with $s=0.00,0.10,0.15,0.22$. Cf. text for explanations
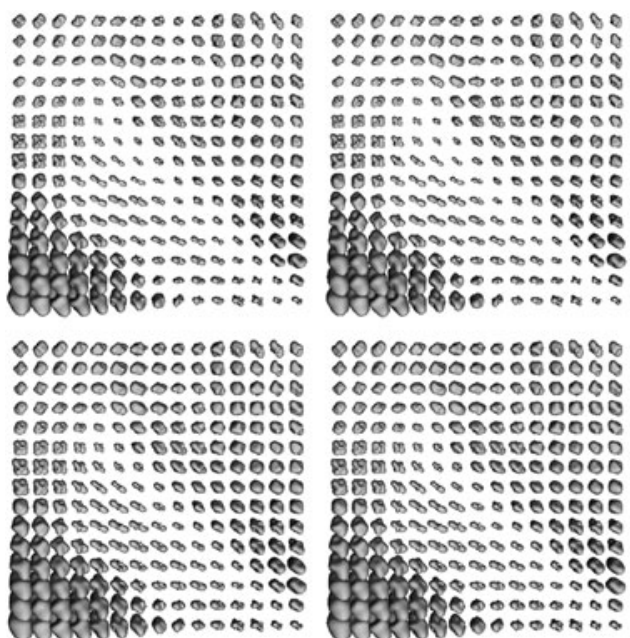

000000000000000 .00000000000000 0000000000000 000000000000 ต 1 क ต ติ Bต 0008000000000

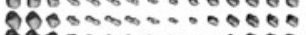
505050508806 CHOR8:0000000 Costro:00:00:00

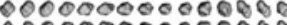
000000000000000 000000000000000 00000000000000 0000000000000 ตำ ตต๊

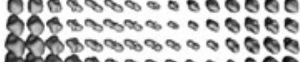
Ut CtSTR

000000000000000 000000000000000 000000000000000 $\therefore 00000000000$ - 0000000000 F 85000000000 8010800000000

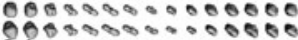

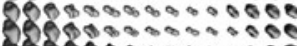
$0,588.05000000$

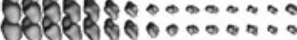

000000000000000 000000000000000 000000000000000 000000000000000 600000000000000 BO 80000000000000 00808000000000
08088900000000 OOBक 000000000 000000000000000 000000000000000

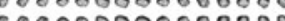

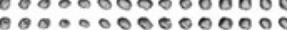
6 * ด B日 008000000000

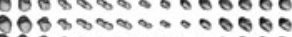

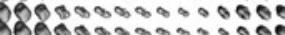
J58 82000000000 ofrses:

\section{0} 00000000000000 000000000000000 000000000000000 통 6อง 28005000000000

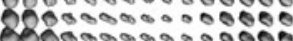
2050500000006 for 280000000000

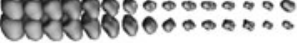
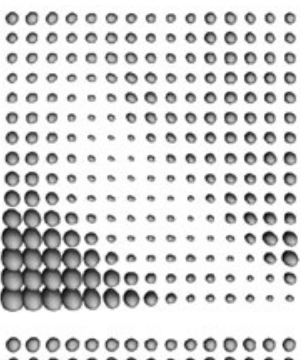
000000000000000 000000000000000 000000000000000

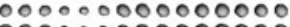
000000000000

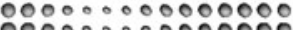
Thob 0.0000000

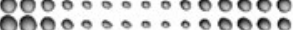
200000000000 $+000000000$ turbo0000000 Atrobo:00:0:00

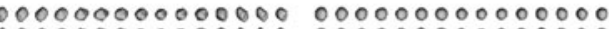

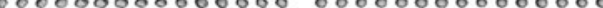

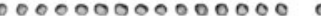
000000000000000000000000000000

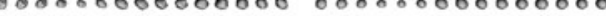
6. 800000000000000000000000000

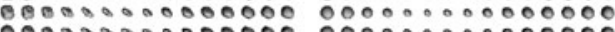

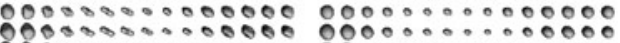

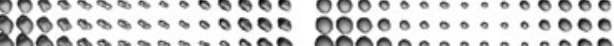

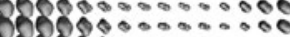
ophes:

(0)

000000000000000000000000000000

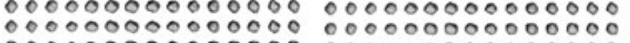
O0000000000000 000000000000000

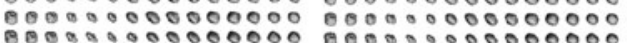
80020000000000 100000000000000

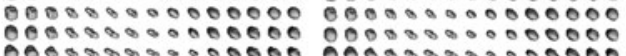
$060 \sin 0000000$ 0003000000000 60\%8

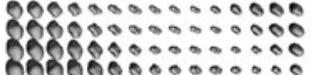

000000000000000 000000000000000 000000000000000 00000000000000 0000000000000 5000000000000

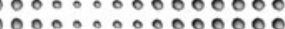
00000000000000 300000.000000 90000000:00000

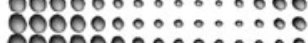

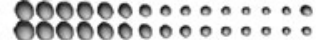

requires a careful balance of order versus enhancement parameter(s). A similar case has been studied in the context of grey-value images (trivial codomain) and spatial deblurring [19] as an extension of traditional image sharpening. ODF enhancement in the HARDI codomain may facilitate fiber tracking.

In all cases, and unlike in previous work, analytical manipulations are carried out directly within the tensorial framework, i.e. without the need to map terms to a spherical harmonic basis. This is especially attractive in the context of a differential geometric rationale. The theory in this paper naturally suggests a shift of paradigm for tractography and connectivity analysis based on a Finslerian rather than Riemannian geometry combined with Tikhonov-like regularization, and provides the basic operational concepts for this.

Several major conceptual issues will need to be addressed in the future in order to apply Finslerian geometry to HARDI problems. One concerns the requirement of positivity, both dictated by the physical nature of diffusion weighted imaging as well as by the axiomatic constraint imposed on the Finsler function [8]. Positivity has been addressed in the case of fourth order representations, cf. Barmpoutis et al. and Ghosh et al. [9, 23]. A second problem concerns the rigorous derivation of the Finsler function itself in terms of HARDI measurement data and the underlying physics of water diffusion. Our suggestion in this paper is of a heuristical nature. A third problem concerns the fact that choices will need to be made when extending methods for tractography and connectivity analysis from the Riemannian to the more general Finslerian framework, for these extensions are not necessarily unique. For instance, the uniqueness of an a priori reasonable (torsion-free) connection compatible with the metric in the Riemannian framework is lost in the Finslerian case, and ambiguities will need to be taken away by physical considerations and/or experimental validation.

Acknowledgements The Netherlands Organisation for Scientific Research (NWO) is gratefully acknowledged for financial support. We thank Erwin Vondenhoff and Mark Peletier for fruitful discussions, and Vesna Prckovska and Paulo Rodrigues for software implementations.

Open Access This article is distributed under the terms of the Creative Commons Attribution Noncommercial License which permits 
any noncommercial use, distribution, and reproduction in any medium, provided the original author(s) and source are credited.

\section{References}

1. Alexander, D.C., Barker, G.J., Arridge, S.R.: Detection and modeling of non-Gaussian apparent diffusion coefficient profiles in human brain data. Magn. Reson. Med. 48(2), 331-340 (2002)

2. Anderson, A.W.: Measurement of fiber orientation distribution using high angular resolution diffusion imaging. Magn. Reson. Med. 54(5), 1194-1206 (2005)

3. Assemlal, H.E., Tschumperlé, D., Brun, L.: Efficient and robust computation of PDF features from diffusion MR signal. Med. Image Anal. 13(5), 715-729 (2009)

4. Astola, L., Florack, L.: Sticky vector fields and other geometric measures on diffusion tensor images. In: Proceedings of the 9th IEEE Computer Society Workshop on Mathematical Methods in Biomedical Image Analysis, Held in Conjunction with the IEEE Computer Society Conference on Computer Vision and Pattern Recognition, Anchorage, Alaska, USA, June 23-28, 2008. IEEE Computer Society, Los Alamitos (2008)

5. Astola, L., Florack, L., ter Haar Romeny, B.: Measures for pathway analysis in brain white matter using diffusion tensor images. In: Karssemeijer, N., Lelieveldt, B. (eds.) Proceedings of the Twentieth International Conference on Information Processing in Medical Imaging-IPMI 2007 Kerkrade, The Netherlands. Lecture Notes in Computer Science, vol. 4584, pp. 642-649. Springer, Berlin (2007)

6. Astola, L.J.: Multi-scale Riemann-Finsler geometry: Applications to diffusion tensor imaging and high resolution diffusion imaging. Ph.D. Thesis, Eindhoven University of Technology, Department of Mathematics and Computer Science, Eindhoven, The Netherlands (2010)

7. Astola, L.J., Florack, L.M.J.: Finsler geometry on higher order tensor fields and applications to high angular resolution diffusion imaging. In: Tai, X.C., Mørken, K., Lysaker, M., Lie, K.A. (eds.) Scale Space and Variational Methods in Computer Vision: Proceedings of the Second International Conference, SSVM 2009, Voss, Norway. Lecture Notes in Computer Science, vol. 5567, pp. 224-234. Springer, Berlin (2009)

8. Bao, D., Chern, S.S., Shen, Z.: An Introduction to RiemannFinsler Geometry. Graduate Texts in Mathematics, vol. 2000. Springer, New York (2000)

9. Barmpoutis, A., Hwang, M.S., Howland, D., Forder, J.R., Vemuri, B.C.: Regularized positive-definite fourth order tensor field estimation from DW-MRI. NeuroImage 45(1 Suppl), S153-162 (2009)

10. Basser, P.J., Mattiello, J., Le Bihan, D.: Estimation of the effective self-diffusion tensor from the NMR spin echo. J. Magn. Reson. 103, 247-254 (1994)

11. Basser, P.J., Mattiello, J., Le Bihan, D.: MR diffusion tensor spectroscopy and imaging. Biophys. J. 66(1), 259-267 (1994)

12. Chen, Y., Guo, W., Zeng, Q., Yan, X., Huang, F., Zhang, H., He, G., Vemuri, B.C., Liu, Y.: Estimation, smoothing, and characterization of apparent diffusion coefficient profiles from high angular resolution DWI. In: Proceedings of the IEEE Computer Society Conference on Computer Vision and Pattern Recognition, Washington DC, USA, June 27-July 2 2004, vol. 1, pp. 588-593. IEEE Computer Society, Los Alamitos (2004)

13. Descoteaux, M., Angelino, E., Fitzgibbons, S., Deriche, R.: Apparent diffusion coefficients from high angular resolution diffusion imaging: Estimation and applications. Magn. Reson. Med. 56(2), 395-410 (2006)
14. Descoteaux, M., Angelino, E., Fitzgibbons, S., Deriche, R.: Regularized, fast, and robust analytical Q-ball imaging. Magn. Reson. Med. 58(3), 497-510 (2007)

15. Descoteaux, M., Savadjiev, P., Campbell, J., Pike, G.B., Siddiqi, K., Deriche, R.: Validation and comparison of analytical Q-ball imaging methods. In: Proceedings of the 4th IEEE International Symposium on Biomedical Imaging: From Nano to Macro, April 12-15, 2007, Washington DC, USA, pp. 1084-1087. IEEE Signal Processing Society, New York (2007). Article no. 4193478

16. Fillard, P., Pennec, X., Arsigny, V., Ayache, N.: Clinical DT-MRI estimation, smoothing, and fiber tracking with log-Euclidean metrics. IEEE Trans. Med. Imag. 26(11), 1472-1482 (2007)

17. Florack, L., Balmashnova, E.: Decomposition of high angular resolution diffusion images into a sum of self-similar polynomials on the sphere. In: Bayakovsky, Y., Moiseev, E. (eds.) Proceedings of the Eighteenth International Conference on Computer Graphics and Vision, GraphiCon'2008, Moscow, Russia, June 23-27, 2008, pp. 26-31. Moscow State University, Moscow (2008). Invited paper

18. Florack, L., Balmashnova, E.: Two canonical representations for regularized high angular resolution diffusion imaging. In: Alexander, D., Gee, J., Whitaker, R. (eds.) MICCAI Workshop on Computational Diffusion MRI, New York, USA, September 10, 2008, pp. 85-96 (2008)

19. Florack, L.M.J., Haar Romeny, B.M.t., Koenderink, J.J., Viergever, M.A.: The Gaussian scale-space paradigm and the multiscale local jet. Int. J. Comput. Vis. 18(1), 61-75 (1996)

20. Fogh Olsen, O., Florack, L., Kuijper, A. (eds.): Deep Structure, Singularities and Computer Vision. Lecture Notes in Computer Science, vol. 3753. Springer, Berlin (2005)

21. Folland, G.B.: How to integrate a polynomial over a sphere. Am. Math. Mont. 108(5), 446-448 (2001)

22. Frank, L.R.: Characterization of anisotropy in high angular resolution diffusion-weighted MRI. Magn. Reson. Med. 47(6), 1083 1099 (2002)

23. Ghosh, A., Descoteaux, M., Deriche, R.: Riemannian framework for estimating symmetric positive definite 4 th order diffusion tensors. In: Proceedings of the 11th International Conference on Medical Image Computing and Computer Assisted Intervention New York, USA, September 6-10, 2008. Lecture Notes in Computer Science, vol. 5241-5242, pp. 858-865. Springer, Berlin (2008)

24. Goh, A., Lenglet, C., Thompson, P.M., Vidal, R.: Estimating orientation distribution functions with probability density constraints and spatial regularity. In: Yang, G.Z., Hawkes, D.J., Rueckert, D., Noble, J.A., Taylor, C.J. (eds.) Proceedings of the 12th International Conference on Medical Image Computing and Computer Assisted Intervention-MICCAI 2009, London, UK, September 20-24, 2009. Lecture Notes in Computer Science, vol. 1, pp. 877885. Springer, Berlin (2009)

25. Hess, C.P., Mukherjee, P., Tan, E.T., Xu, D., Vigneron, D.B.: Q-ball reconstruction of multimodal fiber orientations using the spherical harmonic basis. Magn. Reson. Med. 56, 104-117 (2006)

26. Jensen, J.H., Helpern, J.A., Ramani, A., Lu, H., Kaczynski, K.: Diffusional kurtosis imaging: The quantification of non-Gaussian water diffusion by means of magnetic resonance imaging. Magn. Reson. Med. 53(6), 1432-1440 (2005)

27. Jian, B., Vemuri, B.C., Özarslan, E., Carney, P.R., Mareci, T.H.: A novel tensor distribution model for the diffusion-weighted MR signal. NeuroImage 37, 164-176 (2007)

28. Johnston, T.W.: Cartesian tensor scalar product and spherical harmonic expansions in Boltzmann's equation. Phys. Rev. 120, 1103$1111(1960)$

29. Koenderink, J.J.: The structure of images. Biol. Cybern. 50, 363370 (1984) 
30. Le Bihan, D., Mangin, J.F., Poupon, C., Clark, C.A., Pappata, S., Molko, N., Chabriat, H.: Diffusion tensor imaging: Concepts and applications. J. Magn. Reson. Imag. 13, 534-546 (2001)

31. Lenglet, C., Deriche, R., Faugeras, O.: Inferring white matter geometry from diffusion tensor MRI: Application to connectivity mapping. In: Pajdla, T., Matas, J. (eds.) Proceedings of the Eighth European Conference on Computer Vision Prague, Czech Republic, May 2004. Lecture Notes in Computer Science, vol. 30213024, pp. 127-140. Springer, Berlin (2004)

32. Lenglet, C., Rousson, M., Deriche, R., Faugeras, O.: Statistics on the manifold of multivariate normal distributions: Theory and application to diffusion tensor MRI processing. J. Math. Imag. Vis. 25(3), 423-444 (2006)

33. Liu, C., Bammer, R., Acar, B., Moseley, M.E.: Characterizing non-Gaussian diffusion by using generalized diffusion tensors. Magn. Reson. Med. 51(5), 924-937 (2004)

34. Melonakos, J., Pichon, E., Angenent, S., Tannenbaum, A.: Finsler active contours. IEEE Trans. Pattern Anal. Mach. Intell. 30(3), 412-423 (2008)

35. Misner, C.W., Thorne, K.S., Wheeler, J.A.: Gravitation. Freeman, San Francisco (1973)

36. Özarslan, E., Mareci, T.H.: Generalized diffusion tensor imaging and analytical relationships between diffusion tensor imaging and high angular resolution imaging. Magn. Reson. Med. 50, 955-965 (2003)

37. Özarslan, E., Shepherd, T.M., Vemuri, B.C., Blackband, S.J., Mareci, T.H.: Resolution of complex tissue microarchitecture using the diffusion orientation transform (DOT). NeuroImage 31, 1086-1103 (2006)

38. Pennec, X., Fillard, P., Ayache, N.: A Riemannian framework for tensor computing. Int. J. Comput. Vis. 66(1), 41-66 (2006)

39. Prados, E., Soatto, S., Lenglet, C., Pons, J.P., Wotawa, N., Deriche, R., Faugeras, O.: Control theory and fast marching techniques for brain connectivity mapping. In: Proceedings of the IEEE Computer Society Conference on Computer Vision and Pattern Recognition, New York, USA, June 2006, vol. 1, pp. 1076-1083. IEEE Computer Society, Los Alamitos (2006)

40. Rund, H.: The Hamilton-Jacobi Theory in the Calculus of Variations. Robert E. Krieger Publishing Company, Huntington (1973)

41. Shen, Z.: Lectures on Finsler Geometry. World Scientific, Singapore (2001)

42. Spivak, M.: Differential Geometry, vols. 1-5. Publish or Perish, Berkeley (1975)

43. Stejskal, E.O., Tanner, J.E.: Spin diffusion measurements: Spin echoes in the presence of a time-dependent field gradient. J. Comput. Phys. 42, 288-292 (1965)

44. Tuch, D.S.: Q-ball imaging. Magn. Reson. Med. 52, 1358-1372 (2004)

Luc Florack was born in 1964 in Maastricht, The Netherlands, where he attended primary and secondary school. He received his M.Sc. degree in theoretical physics in 1989, and his Ph.D. degree cum laude in 1993 with a thesis on image structure, both from Utrecht University, The Netherlands. During the period 1994-1995 he was an ERCIM/HCM research fellow at INRIA Sophia-Antipolis, France, and
INESC Aveiro, Portugal. In 1996 he was an assistant research professor at DIKU, Copenhagen, Denmark, on a grant from the Danish Research Council. In 1997 he returned to Utrecht University, were he became an assistant research professor at the Department of Mathematics and Computer Science. In 2001 he moved to Eindhoven University of Technology, Department of Biomedical Engineering, were he became an associate professor in 2002. In 2007 he was appointed full professor at the Department of Mathematics and Computer Science, retaining a parttime professor position at the former department. His research covers mathematical models of structural aspects of signals, images, and movies, particularly multi-scale and differential geometric representations, and their applications to imaging and vision, with a focus on the analysis of tagging magnetic resonance imaging for the heart and diffusion magnetic resonance imaging for the brain, and on biologically motivated models of "early vision".

Evgeniya Balmashnova was born in 1976 in Ust-Ilimsk, Russia. She received her M.Sc. degree in mathematics at Novosibirsk State University, Russia, in 2000. In 2001-2003 she followed the postgraduate program Mathematics for Industry at the Stan Ackermans Institute of Eindhoven University of Technology, The Netherlands. She received her Ph.D. degree in 2007 from Eindhoven University of Technology under supervision of professor Bart ter Haar Romeny and associate professor Luc Florack. She is currently a postdoctoral fellow at the Department of Mathematics and Computer Science at the same university. Her research is focused on medical image analysis, high angular resolution diffusion imaging and diffusion tensor imaging, and in particular, on multi-scale approaches in image analysis.

Laura Astola was born in 1967 in Turku, Finland. She got her high school diploma from Tottori East prefectural high school in Japan. She began her studies in mathematics at Turku University, and subsequently moved to Helsinki University. In April 2000 she finished her M.Sc. thesis on "Computer-Aided Visualization in Mathematics Teaching" and began her Ph.D. studies on geometric analysis in Helsinki University of Technology. She completed her Licenciate's thesis "Lattès Type Uniformly Quasi-Regular Mappings on Compact Manifolds" in February 2009, obtaining the degree of Licenciate of Technology. In February 2006 she started as a Ph.D. student within the Biomedical Image Analysis group of Eindhoven University of Technology. In 2007 she continued her Ph.D. research as a member of CASA (Center for Analysis, Scientific Computing and Applications) at the Department of Mathematics and Computer Science of Eindhoven University of Technology. In January 2010 she obtained her Ph.D. degree under the supervision of professor Luc Florack. The title of her thesis is "Multi-Scale Riemann-Finsler Geometry: Applications to Diffusion Tensor Imaging and High Angular Resolution Diffusion Imaging".

Ellen Brunenberg was born in 1983 in Weert, The Netherlands. She received her M.Sc. degree in Biomedical Engineering from Eindhoven University of Technology, The Netherlands, in 2007. She is currently doing her Ph.D. in Biomedical Engineering at the same university, supported by a TopTalent grant from the Netherlands Organization for Scientific Research, under the supervision of professor Bart ter Haar Romeny. Her research is focused on image analysis for deep brain stimulation in Parkinson's patients. 